Amir H. Zamanipoor Najafabadi, BSc (D)* $*$

Pim B. van der Meer, BSc ${ }^{\ddagger}$

Florien W. Boele, $\mathrm{PhD}^{\S}$ q

Martin J. B. Taphoorn, MD, $\mathrm{PhD}^{\ddagger} \|$

Martin Klein, PhD\#

Saskia M. Peerdeman, MD, PhD**

Wouter R. van Furth, MD, PhD (D)*

Linda Dirven, $\mathrm{PhD}^{\ddagger} \|$

on behalf of the Dutch Meningioma

Consortium

*University Neurosurgical Center Holland Leiden University Medical Center, Haaglanden Medical Center and Haga Teaching Hospital, Leiden and The Hague, the Netherlands; ${ }^{\ddagger}$ Department of Neurology, Leiden University Medical Center, Leiden, the Netherlands; ${ }^{\S}$ Leeds Institute of Medical Research at St James's, St James's University Hospital, Leeds, United Kingdom; "Leeds Institute of Health Sciences, Faculty of Medicine and Health, University of Leeds, Leeds, United Kingdom; $\|_{\text {Department }}$ of Neurology, Haaglanden Medical Center, The Hague, the Netherlands; \#Brain Tumor Center Amsterdam at Amsterdam University Medical Centers, location VUmc, Amsterdam, the Netherlands; ** Department of Neurosurgery, Amsterdam University Medical Centers, location VUmc, Amsterdam, the Netherlands

Parts of the results described in this manuscript were presented at the 2018 Society for Neuro-Oncology (SNO) conference in New Orleans, Louisiana, as an oral presentation on 17 November 2018. In addition, parts of the results were presented at the 2018 European Association of Neuro-Oncology(EANO) conference in Stockholm, Sweden, as an oral presentation on 13 October 2018.

\section{Correspondence:}

Amir H. Zamanipoor Najafabadi, BSC, Department of Neurosurgery and Neurology,

Leiden University Medical Center postal zone J11-R, Albinusdreef 2 , 2333ZA Leiden, the Netherlands. Email: amir@lumc.nl

Twitter: @Amir_Zamanipoor

Received, November 17, 2019

Accepted, June 8, 2020.

(c) Congress of Neurological Surgeons 2020.

This is an Open Access article distributed under the terms of the Creative Commons Attribution-NonCommercialNoDerivs licence (http://creative commons.org/licenses/by-nc-nd/4.0/), which permits non-commercial reproduction and distribution of the work, in any medium, provided the original work is not altered or transformed in any way, and that the work is properly cited. For commercial re-use, please contact journals.permissions@oup.com

\title{
Long-Term Disease Burden and Survivorship Issues After Surgery and Radiotherapy of Intracranial Meningioma Patients
}

BACKGROUND: Many intracranial meningioma patients have an impaired health-related quality of life (HRQoL) and neurocognitive functioning up to $4 \mathrm{yr}$ after intervention. OBJECTIVE: To assess the long-term ( $\geq 5 \mathrm{yr}$ ) disease burden of meningioma patients. METHODS: In this multicenter cross-sectional study, patients $\geq 5 \mathrm{yr}$ after intervention (including active magnetic resonance imaging (MRI) surveillance) were included and assessed for HRQoL (Short-Form Health Survey 36), neurocognitive functioning (neuropsychological assessment), anxiety and depression (Hospital Anxiety and Depression Scale), and work productivity (Short Form-Health and Labour Questionnaire). Multivariable and propensity score regression analyses were used to compare patients and controls, and different treatment strategies corrected for possible confounders. Clinically relevant differences were reported.

RESULTS: At a median of $9 \mathrm{yr}$ follow-up after intervention, meningioma patients $(\mathrm{n}=190)$ reported more limitations due to physical (difference 12.5 points, $P=.008$ ) and emotional (13.3 points, $P=.002$ ) health problems compared with controls. Patients also had an increased risk to suffer from anxiety (odds ratio [OR]: $2.6,95 \% \mathrm{Cl}: 1.2-5.7$ ) and depression (OR: 3.7, 95\% Cl: 1.3-10.5). Neurocognitive deficits were found in $43 \%$ of patients. Although postoperative complications, radiotherapy, and reresection were associated with worse verbal memory, attention, and executive functioning when compared to patients resected once, the only clinically relevant association was between reresection and worse attention $(-2.11,95 \% \mathrm{Cl}:-3.52$ to -0.07$)$. Patients of working age less often had a paid job (48\%) compared with the working-age Dutch population (72\%) and reported more obstacles at work compared with controls.

CONCLUSION: In the long term, a large proportion of meningioma patients have impaired $\mathrm{HRQ}$ L, neurocognitive deficits, and high levels of anxiety or depression. Patients treated with 1 resection have the best neurocognitive functioning.

KEY WORDS: Meningioma, Quality of life, Cognitive function, Anxiety, Depression

Neurosurgery 0:1-10, 2020 DOI:10.1093/neuros/nyaa351 www.neurosurgery-online.com

$\mathbf{M}$ eningioma accounts for $37 \%$ of all primary brain tumors. ${ }^{1-3}$ Morbidity of intracranial meningiomas is primarily due to compression of brain tissue and cranial nerves, or treatment-related complications (eg, hemorrhage). ${ }^{3}$ Primary treatment for these tumors consists of surgery, with in selected cases first-line or adjuvant radiotherapy, resulting in a 10 -year relative survival of $82 \%$ for World Health Organization (WHO) grade I meningioma. ${ }^{2,4,5}$

ABBREVIATIONS: EORTC QLQ-BN20, European Organization for Research and Treatment of Cancer quality of life questionnaire brain specific module; HADS, Hospital Anxiety and Depression Scale; HRQoL, health-related quality of life; MAAS, Maastricht Aging Study; MCID, minimal clinically important differences; MRI, magnetic resonance imaging; OR, odds ratio; SD, standard deviation; SF-HLQ, Short Form-Health and Labour Questionnaire; SF-36, Short-Form Health Survey 36; WHO, World Health Organization

Supplemental digital content is available for this article at www.neurosurgery-online.com. 
One might expect that after decompression of central nervous tissue, symptoms are resolved and functioning returns to normal eventually. Historically, long-term meningioma survivors ( $\geq 5 \mathrm{yr}$ after intervention) who lived through the diagnosis and treatment of a meningioma were often considered "cured." $3,6,7$ However, it is known from cancer populations that the experience of living beyond tumor and treatment entails considerable lifelong physical, cognitive, and psychological issues (eg, neurocognitive impairments and disrupted social roles), which often differ from the acute complications patients experience during diagnosis and treatment (eg, impaired physical function due to paresis). ${ }^{8,9}$

Although it is known that surgery and radiotherapy might improve health-related quality of life (HRQoL) and cognitive function in the first year, recent studies have shown that up to a median of $4 \mathrm{yr}$ after intervention, meningioma patients may still suffer from impaired HRQoL and neurocognitive functioning, and increased anxiety and depression. ${ }^{7,10,11}$ Although data on long-term effects of meningioma and its treatment on these outcomes are lacking, studies in low-grade glioma suggest that some impairments and deficits only manifest $5 \mathrm{yr}$ beyond treatment. ${ }^{12}$ Moreover, the impact on societal participation in terms of work productivity is currently unknown.?

Thus, we aimed to assess the long-term ( $\geq 5 \mathrm{yr}$ after their intervention, ie, last antitumor treatment or initiation of active magnetic resonance imaging [MRI] surveillance) disease burden of meningioma patients in terms of HRQoL, anxiety and depression, neurocognitive functioning, and work productivity. We also assessed if these outcomes were affected by the type of treatment received. Better knowledge of long-term survivorship issues in meningioma patients will help to manage patients' expectations and design long-term meningioma care plans, tailored to patient's physical, psychological, and social needs.

\section{METHODS}

\section{Participants}

In this multicenter cross-sectional study, consecutive meningioma patients were included if the end of the primary antitumor treatment was at least $5 \mathrm{yr}$ prior to recruitment, or in case of active MRI surveillance, at least $5 \mathrm{yr}$ after diagnosis. Eligible patients had to be $18 \mathrm{yr}$ or older, with a histologically confirmed WHO grade I or grade II meningioma in case of surgery and an MRI-based clinically suspected meningioma in case of radiotherapy or active MRI surveillance. Consecutive patients were recruited from the neurosurgery, neurology, and radiation oncology outpatient clinics of 2 academic hospitals and 1 large nonacademic teaching hospital between July 2016 and April 2019. All eligible patients were approached for this study via a letter signed by a member of their treatment team. Patients were excluded if they had a history of whole brain radiotherapy, were diagnosed with neurofibromatosis type II or any neurodegenerative disease, or had insufficient mastery of the Dutch language.

Informal caregivers of participating meningioma patients were included for comparison of HRQoL, anxiety and depression, and work productivity and were eligible for participation if they were a spouse, family member, or close friend to the patient, $18 \mathrm{yr}$ or older, and provided the majority of emotional or physical support to the patient as reported by the patient. It was not possible to include an informal caregiver for every patient, as some patients were not able to identify an informal caregiver motivated to participate in the study.

\section{Procedures}

This cross-sectional study was approved by the medical ethical committees of all participating centers (NL54866.029.15), and participants provided informed consent before study procedures. Both questionnaires and neurocognitive assessment were administered once on the same day, at least $5 \mathrm{yr}$ after their last meningioma treatment. Hence, there is variation in the follow-up length between patient's last meningioma treatment and moment of study participation. Clinical information on tumor and treatment was obtained from the medical records, while sociodemographic information about patients and controls was obtained through a structured interview at the beginning of the assessments.

\section{Questionnaires}

Patients completed questionnaires measuring HRQoL consisting of the Short-Form Health Survey 36 (SF-36) and European Organization for Research and Treatment of Cancer quality of life questionnaire, brain specific module (EORTC QLQ-BN20). In addition, patients completed the Hospital Anxiety and Depression Scale (HADS), and Short FormHealth and Labour Questionnaire (SF-HLQ) measuring work productivity. Informal caregivers completed the same questionnaires, except for the EORTC QLQ-BN20 (Supplemental Digital Content 1).

\section{Neuropsychological Assessment}

A comprehensive battery of neuropsychological tests was administered by trained research nurses or research assistants and consisted of the Auditory Verbal Learning Test, Concept Shifting Test, Memory Comparison Test, Categoric Word Fluency Test, Digit-Symbol Substitution Test, and the Stroop Color-Word Test. Based on these tests, scores for the following neurocognitive domains were calculated: executive functioning, verbal memory, working memory, psychomotor functioning, information processing speed, and attention (Supplemental Digital Content 1).

\section{Statistical Analysis}

A description of the sample size calculation is provided in Supplemental Digital Content 2. SF-36 and EORTC QLQ-BN20 scores were presented for each domain or scale/item, respectively, ranging from 0 to 100 , with higher scores representing better HRQoL (SF-36), or more symptomatology (QLQ-BN20). Total scores for both anxiety and depression as measured with the HADS range from 0 to 21 and were classified into no (scores: 0-7), borderline (scores: 8-10), and severe anxiety or depression (scores: 11-21). ${ }^{13}$ Work productivity was measured as having a paid job or not and experienced difficulties at work on 6 items. ${ }^{14}$ Unadjusted crude scores on the SF-36, EORTC QLQ-BN20, and HADS for both patients and controls (ie, informal caregivers) are presented in bar graphs.

Data on HRQoL (SF-36 only), anxiety and depression, and work productivity were compared between meningioma patients and informal caregivers, corrected for known confounders (ie, age, gender, education level, and comorbidity) using multivariable regression analysis. ${ }^{10,12}$ As a sensitivity analysis, data on HRQoL as measured with the SF-36 was also compared between meningioma patients and published normative 
data using a one-sample $t$-test. For the EORTC QLQ-BN20 data, we performed a one-sample $t$-test to compare meningioma data with baseline data (ie, after surgery but before further antitumor treatment) of glioblastoma patients from the AVAglio trial. ${ }^{15}$ This comparison with the most common primary malignant brain tumor was done to put disease-specific HRQoL into context. As minimal clinically important differences (MCIDs) were not known for the used instruments in brain tumor patients specifically, we used MCIDs previously established for other patient groups. MCIDs was set on 10 points for scales/items of the EORTC QLQ-BN20. ${ }^{16}$ Similarly, we set the MCID for the SF-36 domains also at 10 points, as the majority of reported MCID's for the different domains were $<10$ points. ${ }^{17}$ For the SF-36 mental and physical component scales, MCIDs were set at 4.6 points and 3.0 points, resepectively. ${ }^{18}$ Furthermore, for calculation of $\mathrm{z}$-scores for each neurocognitive domain, means and standard deviations (SDs) from a reference sample from the Maastricht Aging Study (MAAS); large longitudinal study on the psychological and biological determinants of cognitive aging) were used, matched on group level for age, gender, and educational level. ${ }^{19}$ Per domain, differences in $\mathrm{z}$-scores greater than -1.5 were considered clinically relevant. ${ }^{20}$ In addition, meningioma patients of working age were compared with net average working-age Dutch population (source: Statistics Netherlands) for comparison of the percentage patients with a paid job. $^{21}$

The effects of surgery and radiotherapy were compared for those SF$36 \mathrm{HRQoL}$ and neurocognitive functioning domains on which patients scored clinically relevant lower compared with controls, limiting the number of statistical tests performed. Propensity score regression analysis was used (see Supplemental Digital Content 2 for details) to adjust for potentially relevant confounders (eg, age, tumor size, tumor location, and Simpson grade)..$^{7,22}$

A nonresponders analysis was performed comparing important clinical and sociodemographic characteristics between participating meningioma patients and patients who chose not to participate. For all statistical tests, SPSS 23 (IBM, Armonk, New York) was used, and P less than .05 was considered statistically significant.

\section{RESULTS}

\section{Demographics}

A total of 190 patients (female: $n=149,78 \%$ ) were included with a median follow-up since intervention of $9 \mathrm{yr}$ (IQR: 712 yr) (Table, Figure 1). Patients were on average 63 yr old (SD: 12). Tumors were located on the skull base in 92 patients $(48 \%)$, the cerebral convexity in 93 patients $(49 \%)$, and the optic nerve sheets or intraventricularly in 5 patients $(3 \%)$. The majority of surgically treated meningioma was classified as WHO grade I $(88 \%)$. Surgery was the primary choice of treatment in 168 (88\%) patients, of whom 63 suffered from any postoperative complication, such as cranial nerve deficits $(n=8)$ or cerebrospinal fluid leak $(n=8)$. A total of $26(14 \%)$ were treated with adjuvant radiotherapy and $13(7 \%)$ with reresection. Primary radiotherapy was limited to $10(5 \%)$ patients with anatomically complicated skull base tumors. A total of 12 patients (6\%) were solely followed with active MRI surveillance without any antitumor treatment. Patient-and tumor-related characteristics in each treatment group are presented in Supplemental Digital Content 3. A total of 129 informal caregivers of participating meningioma patients were included and data from 151 participants of the MAAS study. Nonresponder analysis showed that participating and not participating meningioma patients were similar on important sociodemographic and clinical characteristics, except for age, as not participating patients were slightly older (Supplemental Digital Content 4).

\section{Health-Related Quality of Life (HRQoL)}

After correction for confounders, patients had clinically relevant lower HRQoL scores than controls on 2 of the 8 SF-36 domains: role limitations due to physical health problems (corrected difference 12.5 points, $P=.008$ ), and role limitations due to emotional health problems $(13.3, P=.002)$. In addition, they scored statistically significantly, but not clinically relevant, lower on 2 additional domains and 1 component score: social functioning $(7.4, P=.008)$, vitality $(7.1, P=.016)$, and the mental component score $(3.8, P=.005)$. No differences were found for the other 4 domains and physical component score (Figure 2). In the sensitivity analysis comparing meningioma patients with normative data without correction for confounders, patients had clinically relevant lower scores on 1 domain and 1 component score: role limitations due to physical health problems (uncorrected difference 12.2, $P<.001$ ), and the physical component score $(5.0, P<.001)$. They scored statistically significant, but not clinically relevant, lower on 3 additional domains: physical functioning $(5.4, P=.004)$, general health (7.2, $P<.001)$, and social functioning (5.6, $P=.005)$ (Supplemental Digital Content 5). Comparing meningioma patients with glioblastoma patients after surgery but naive to chemotherapy and radiotherapy, we found that meningioma patients had statistically similar scores on $4 / 11$ EORTC QLQ-BN20 scales/items, showing impaired HRQoL: visual disorder (difference: $2.5, P=.078$ ), communication deficit $(-1.8, P=.291)$, headache $(2.8, P=.296)$, and hair loss $(2.3$, $P=.101)$. The differences were not clinically relevant for these scales/items or any of the other scales/items except future uncertainty, for which glioblastoma patients reported more uncertainty (Figure 3).

\section{Anxiety and Depression}

Patients suffered more frequently from borderline $(8 \%, \mathrm{n}=15)$ and severe $(14 \%, \mathrm{n}=27)$ anxiety, compared with controls (borderline: $6 \%, \mathrm{n}=8$; severe: $3 \%, \mathrm{n}=4$; overall $P=.047$, Figure 4). Patients also suffered more frequently from borderline $(9 \%, \mathrm{n}=16)$ and severe $(8 \%, \mathrm{n}=15)$ depression, compared with controls (borderline: $3 \%, \mathrm{n}=4$; severe: $2 \%, \mathrm{n}=2$; overall $P=.099$, Figure 4). Compared to controls, patients had an increased risk to develop borderline or severe anxiety (odds ratio (OR): 2.6, 95\% CI: 1.2-5.7) and borderline or severe depression (OR: 3.7, 95\% CI: 1.3-10.5) after correction for confounders. 
TABLE. Sociodemographic and Clinical Characteristics of Meningioma Patients and Controls

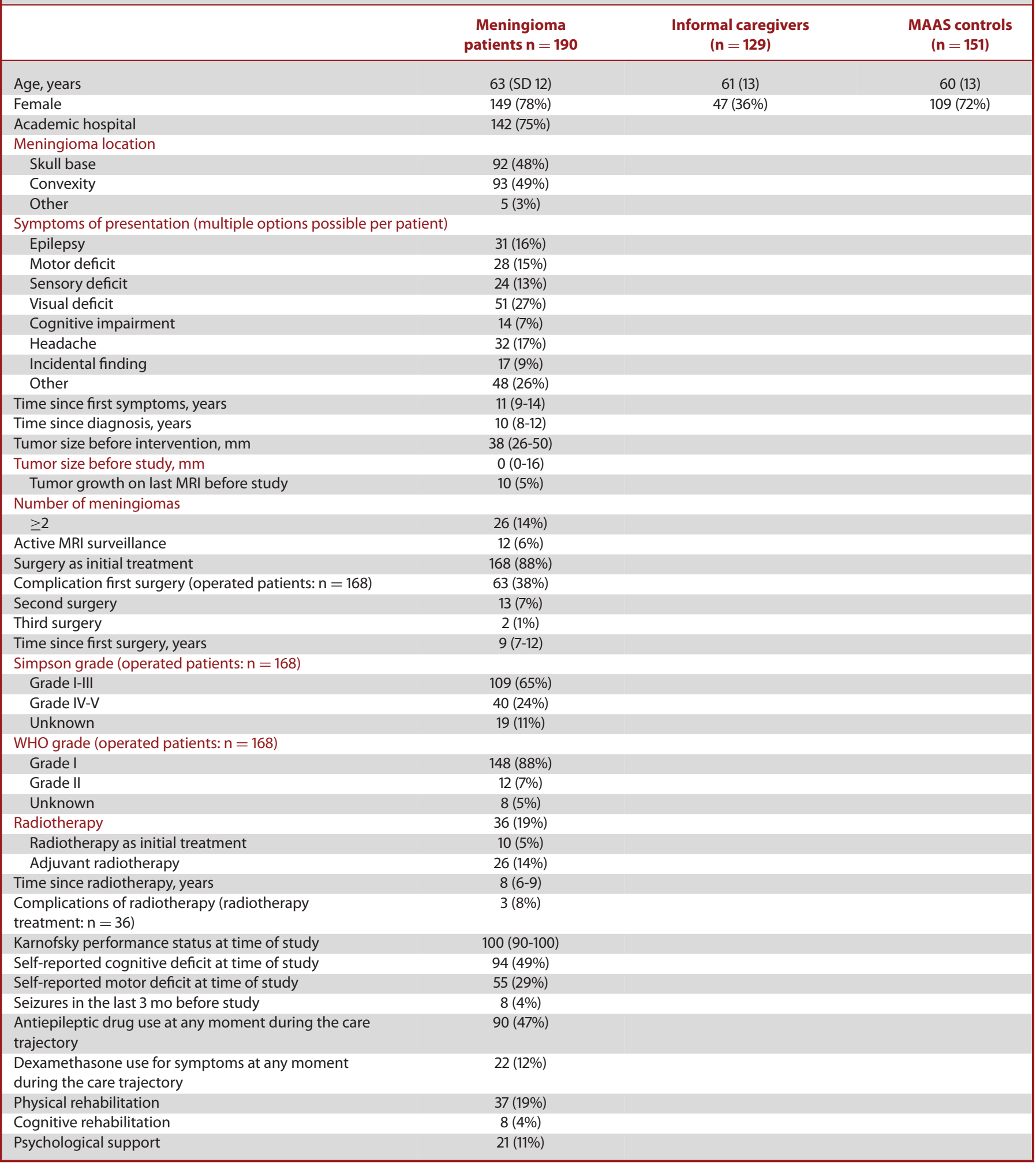




\begin{tabular}{|c|c|c|c|}
\hline & $\begin{array}{c}\text { Meningioma } \\
\text { patients } n=190\end{array}$ & $\begin{array}{l}\text { Informal caregivers } \\
\qquad(n=129)\end{array}$ & $\begin{array}{l}\text { MAAS controls } \\
\quad(n=151)\end{array}$ \\
\hline Other supportive care & $10(5 \%)$ & & \\
\hline \multicolumn{4}{|l|}{ Education level } \\
\hline Primary/secondary & $40(21 \%)$ & $14(11 \%)$ & $58(38 \%)$ \\
\hline Tertiary: technical/vocational & $85(45 \%)$ & $55(43 \%)$ & $49(32 \%)$ \\
\hline Academic & $59(31 \%)$ & $57(44 \%)$ & $45(30 \%)$ \\
\hline Not provided & $6(3 \%)$ & $3(2 \%)$ & \\
\hline \multicolumn{4}{|l|}{ Charlson Comorbidity Index } \\
\hline 0 & $127(67 \%)$ & $88(68 \%)$ & \\
\hline $1 \geq$ & $63(23 \%)$ & $41(32 \%)$ & \\
\hline Right-handed & $147(77 \%)$ & 92 (71\%) & \\
\hline
\end{tabular}

Note. Informal caregivers and controls from the MAAS study.

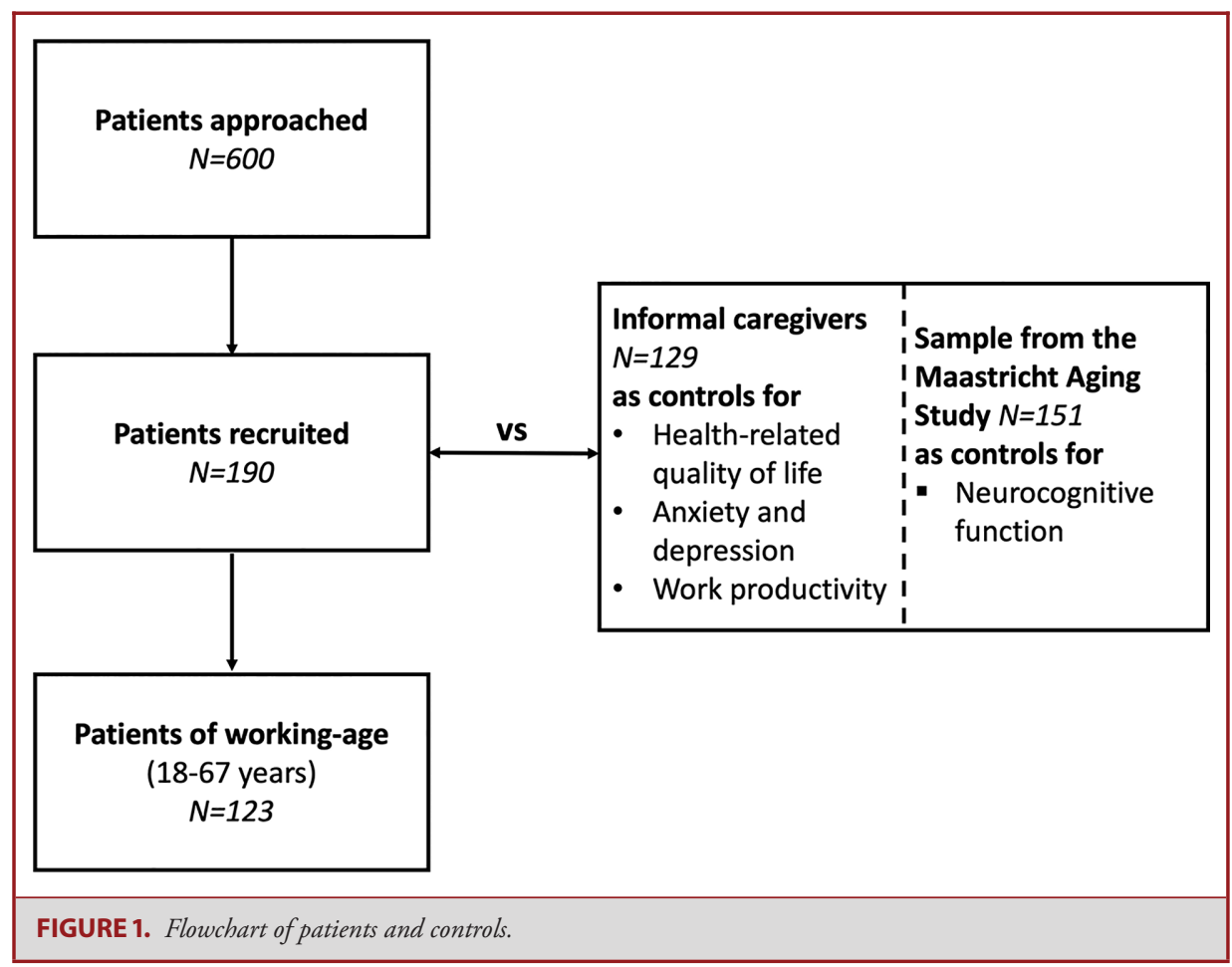

\section{Neurocognitive Functioning}

A total of $43 \%(n=82)$ of patients suffered from a clinically relevant neurocognitive deficit in at least 1 of the 6 measured domains, most often in the domains of information processing speed $(\mathrm{n}=51,27 \%)$ and attention $(\mathrm{n}=44,23 \%)$ (see Figure 5 for all domains). Furthermore, 47 (25\%) patients suffered from a clinically relevant impairment in at least 2 domains, $32(17 \%)$ patients in 3 domains, $22(12 \%)$ patients in 4 domains, $20(11 \%)$ patients in 4 domains, and 7 (4\%) patients in all 6 domains.

\section{Work Productivity}

Out of 190 meningioma patients, 123 (65\%) were aged between $18 \mathrm{yr}$ and $67 \mathrm{yr}$ and considered to be of working age. At the time of assessment, 50\% (62/123) of meningioma patients had a paid job, compared with $72 \%$ of the net average working-age Dutch population $(P<.001)$. Reported reasons to not have a paid job were being a homemaker (female patients $15 \%$, male patients $0 \%$ ) or poor health condition (both male and female patients: $24 \%$ ). More patients reported obstacles at work $(46 \%)$ than controls $(17 \%, P=.005)$. The following 

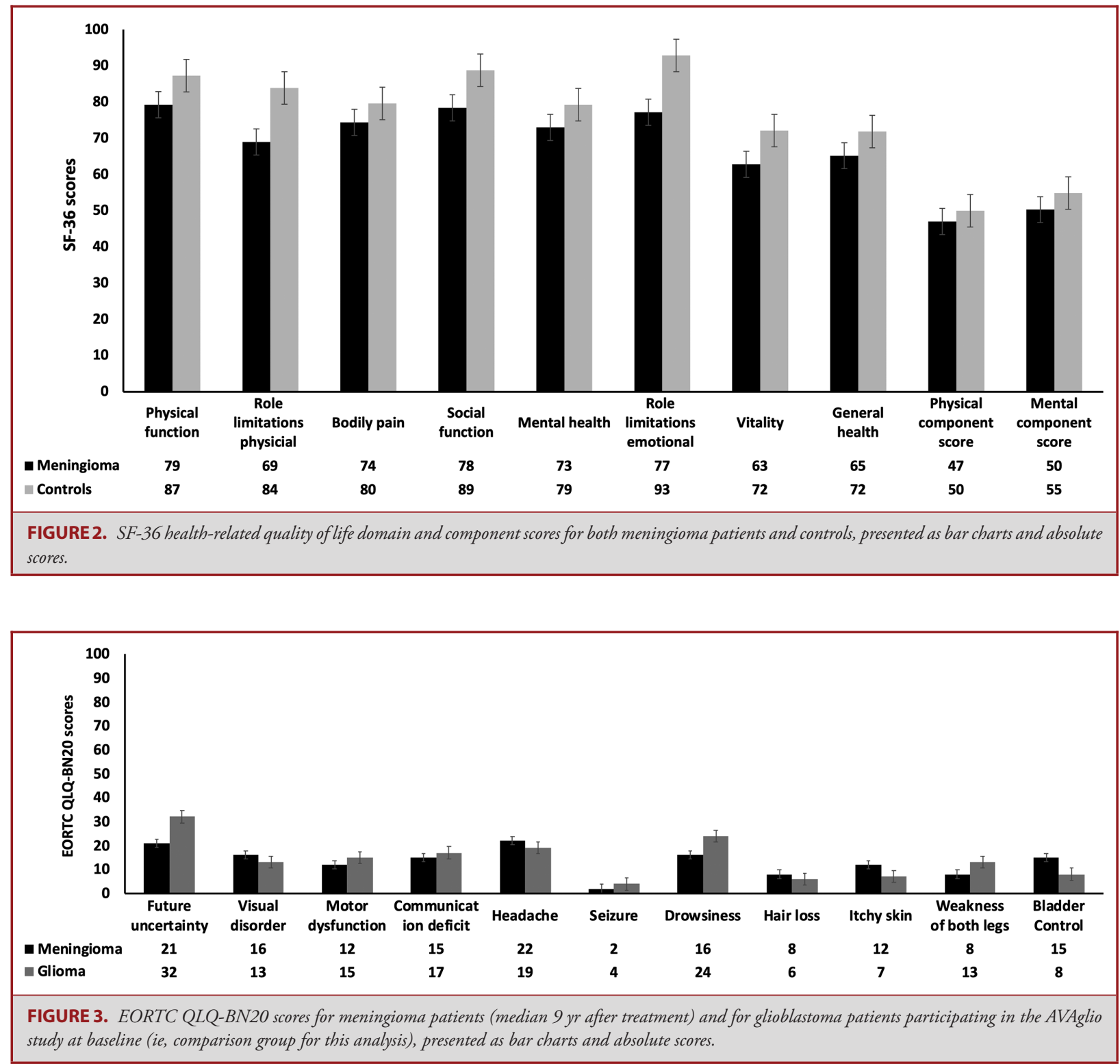

problems at work were reported to occur sometimes to always (Figure 6): impaired concentration (74\%), slower work pace (78\%), feeling isolated (22\%), delaying work (67\%), the need for someone to take over their work (42\%), and problems to make decisions (59\%).

\section{Impact of Surgery and Radiotherapy on HRQoL and Neurocognition}

Patients treated with first line surgery or radiotherapy did not score significantly different on HRQoL or neurocognitive functioning compared to patients followed with active MRI surveillance (Supplemental Digital Contents 6-11). However, comparing surgery with radiotherapy as first-line treatment showed that patients treated with radiotherapy scored significantly worse on verbal memory $(-0.99,95 \%$ CI -1.78 to $-0.20)$. Similarly, patients receiving additional radiotherapy after surgery scored worse on verbal memory $(-0.45,95 \% \mathrm{CI}-0.86$ to -0.03$)$ compared with patients solely treated by surgery. Patients who suffered from a complication of their first surgery scored worse on attention $(-0.78,95 \% \mathrm{CI}-1.42$ to -0.14$)$ compared with those without complications. Especially the need for a second resection for residual tumor or recurrence resulted in worse scores in executive functioning $(-0.92,95 \% \mathrm{CI}-1.78$ to -0.07$)$, verbal memory $(-0.66,95 \% \mathrm{CI}-1.25$ to -0.08$)$, and attention $(-2.11,95 \% \mathrm{CI}-3.52$ to -0.71$)$ compared with patients who only needed a single resection. Except for attention 

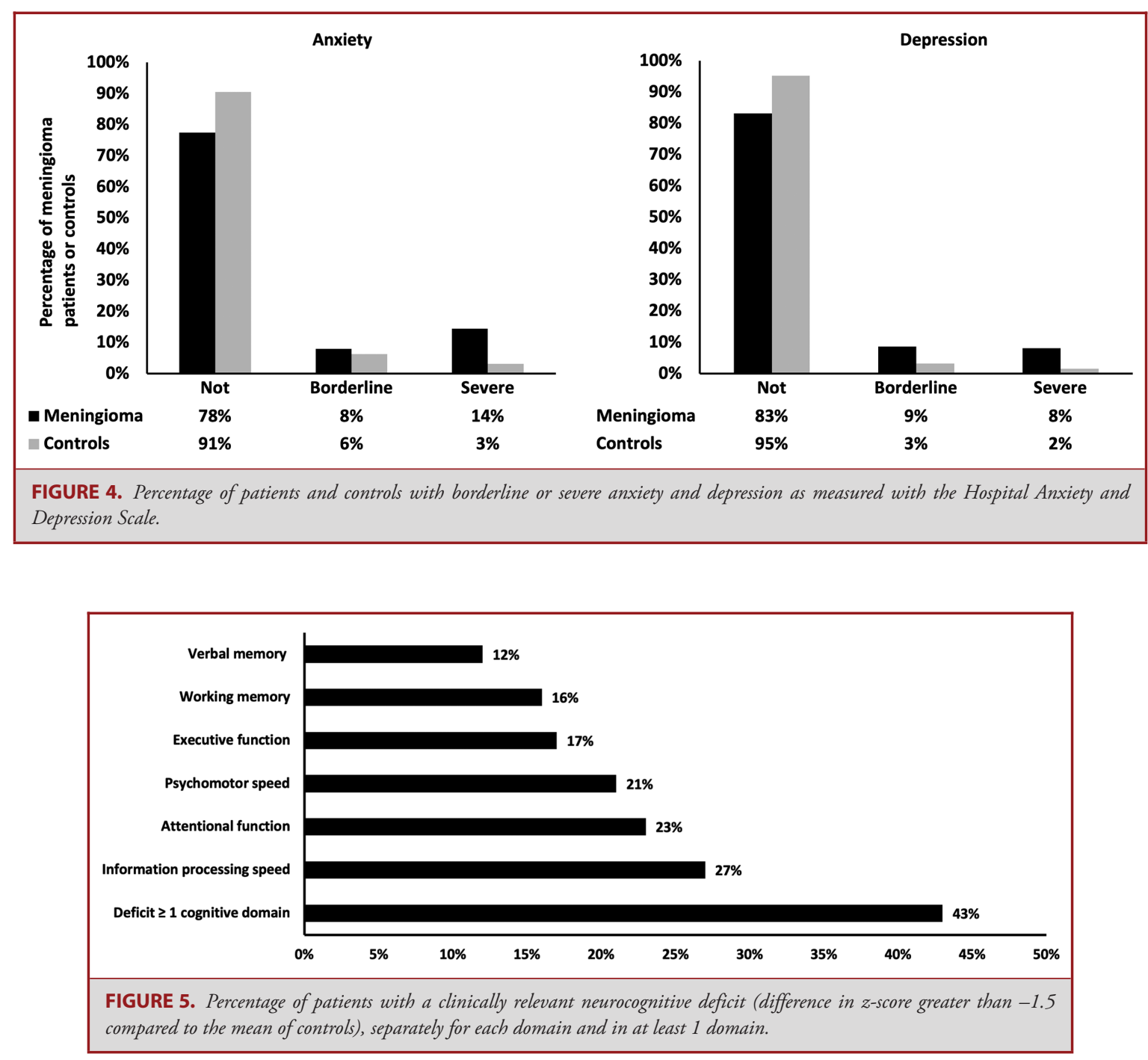

in those patients needing a second resection, differences were not clinically relevant.

\section{DISCUSSION}

\section{Key Results}

Although most meningioma patients have a benign WHO grade I tumor with an associated near-normal life expectancy and are often considered cured after intervention, our results show firm evidence that patients still suffer from a significant disease burden even after a median follow-up of 9 yr. Many patients suffer from clinically relevant impaired HRQoL and neurocognitive functioning, higher levels of anxiety and depression, and lower levels of work productivity. The type of treatment also impacted outcomes; patients who received 1 single resection had better neurocognitive functioning compared with patients who experi- enced surgical complications or were treated with (additional) radiotherapy or who needed a reresection.

\section{Limitations}

Due to the observational cross-sectional design of this study, no conclusions can be drawn on possible improvement or deterioration after treatment and the results might suffer from confounding and bias. Especially for the comparison between patients treated with surgery or radiotherapy as first-line treatment, selection bias might have affected the results, as radiotherapy is often reserved for patients who are older, suffer from comorbidities, or with a complicated anatomic tumor location. To reduce the impact of confounding on our results, particularly when analyzing the cohort, we corrected our analyses for multiple confounders using multivariable and propensity scores regression analysis. Furthermore, we included a limited number of patients with active MRI surveillance or radiotherapy 


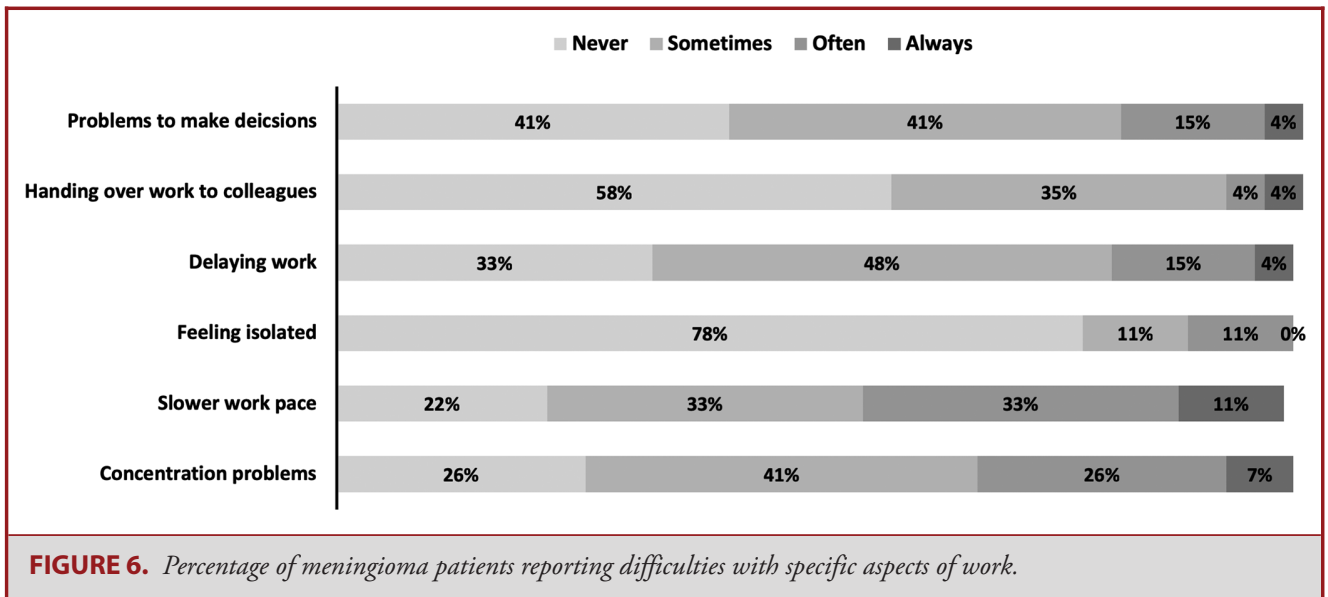

as only treatment. Although radiotherapy is expected to have a negative impact on outcomes in the long term, the number of patients included with radiotherapy was too small to detect small meaningful differences. In addition, we might miss meningiomaspecific HRQoL issues as we used the widely implemented SF36, which enabled comparisons with other patient groups. There is no validated meningioma-specific HRQoL instrument that we could have used. ${ }^{23}$ Moreover, as brain tumor-specific MCIDs are not available for the questionnaires used, we used more conservative MCIDs based on other patient populations. Hence, the presented results might be on the more conservative side. Lastly, we used both informal caregivers and normative data as controls for HRQoL. As informal caregivers are indirectly affected by the disease course of their loved ones but do not suffer directly from the same physical and neurological consequences, we were able to more accurately assess the impact of the tumor and its treatment. Results of both comparisons were fairly similar, showing that compared with informal caregivers or normative data, patients scored clinically relevant lower on several domains/component scores of the SF-36.

\section{Interpretation}

Existing frameworks for survivorship issues describe that while in the acute phase of diagnosis and treatment, bodily impairments can be expected, on the longer term patients primarily experience disruptions of their social roles. ${ }^{7,9}$ Indeed, we found that patients on the longer term reported clinically relevant more role limitations due to physical and emotional health problems, whereas previous studies reported impairments in cognitive and physical functioning at a median of 6 mo and $4 \mathrm{yr}$ after surgery.7,24 Remarkably, we found that patients with a benign meningioma after long-term follow-up had similar HRQoL scores compared with chemotherapy and radiotherapy naive glioblastoma patients. Although the 2 groups are not comparable in terms of follow-up length after treatment initiation, glioblastoma patients are often considered having HRQoL impairments. ${ }^{25}$ To put the results in context of major surgery in non-CNS related conditions, which may also have a huge long-term impact on patients' functioning and well-being, meningioma patients reported lower physical and mental HRQoL than similarly aged patients who received coronary artery bypass graft surgery, ${ }^{26}$ and lower mental but better physical HRQoL compared with patients who received a total hip replacement. ${ }^{27}$ No neuropsychological impairments in meningioma patients have been reported up to a median of $3 \mathrm{yr}$ after intervention. ${ }^{10,28}$ In low-grade glioma patients, these deficits might only become apparent after more than $10 \mathrm{yr}$ of follow-up. ${ }^{12}$ Indeed, we found that neurocognitive deficits were present in over $40 \%$ of meningioma patients. The limited published data on anxiety and depression describe that approximately $10 \%$ to $15 \%$ of meningioma patients suffer from severe depression or anxiety, respectively, both before and 6 mo after surgery. ${ }^{11,29}$ It seems this percentage does not reduce over time, as we found percentages of patients at risk for severe depression or anxiety of $8 \%$ and $14 \%$, respectively. Furthermore, we found that patients less often have a paid job than the age-matched Dutch population, because they were identified as a homemaker (female patients $15 \%$, male patients $0 \%$ ) or due to poor health condition (both male and female patients: 24\%). Compared with patients with prolactinoma, another benign intracranial lesion primarily affecting women, meningioma patients of working age had less often a paid job (meningioma patients: $50 \%$; prolactinoma patients: $80 \%){ }^{27}$ Comparably, female breast cancer patients do not have a paid job due to their health issues and less often because they were homemakers. ${ }^{28,29}$ Although not measured over time, we found that patients who were treated by a single surgery reported better HRQoL and neurocognitive functioning compared with patients treated primarily with radiotherapy or additional radiotherapy or reresection. Previous longitudinal studies in meningioma patients reported improved but not normalized neurocognitive functioning and HRQoL after surgery. ${ }^{28,30}$ Patients treated with radiotherapy showed improvement in HRQoL in the first 6 mo after irradiation, with deterioration to preradiotherapy levels after $2 \mathrm{yr}^{31}$ Only $1(\mathrm{n}=18)$ study has compared the effects 
of postoperative radiotherapy in meningioma patents, reporting no differences in HRQoL. ${ }^{32}$ However, the limited follow-up of $1 \mathrm{yr}$ hampered assessment of possible long-term neurotoxicity of radiotherapy.

\section{Generalizability}

We believe that our results are generalizable, as the number of missing data was very limited (all assessments were performed on a single day), patients were recruited from both academic and nonacademic hospitals in the Netherlands, and because our nonresponder analysis showed that our study population was representative of the general meningioma population. Generalizability to other countries might be hampered due to differences in health care settings and the impact of cultural differences on outcomes such as HRQoL.

\section{CONCLUSION}

Although the continued improvement in surgical and radiotherapeutic techniques for meningioma treatment has resulted in an increase in long-term survivors, little was known about the survivorship issues of these patients. The results of this study show that the longer term disease burden is considerable. This information is of importance to properly inform health care providers and patients on the long-term sequelae of tumor and treatment. This is relevant for proper expectation management, as well as to develop care plans for long-term survivors, focusing on the identified longer term impairments. Lastly, the results of this study can be used as a benchmark for comparison of multiple patient-centered outcomes in the long term when evaluating new treatment modalities. ${ }^{33}$ Possible determinants for the long-term disease burden are an important topic and should be explored in more detail in future studies.

\section{Funding}

This study did not receive any funding or financial support.

\section{Disclosures}

The authors have no personal, financial, or institutional interest in any of the drugs, materials, or devices described in this article. Mr Zamanipoor Najafabadi was supported by a personal $\mathrm{MD} / \mathrm{PhD}$ grant of the Leiden University Medical Center.

\section{REFERENCES}

1. Ostrom QT, Gittleman H, Fulop J, et al. CBTRUS statistical report: primary brain and central nervous system tumors diagnosed in the united states in 2008-2012. Neuro Oncol. 2015;17(suppl 4):iv1-iv62.

2. Ostrom QT, Gittleman H, Truitt G, Boscia A, Kruchko C, Barnholtz-Sloan JS. CBTRUS statistical report: primary brain and other central nervous system tumors diagnosed in the united states in 2011-2015. Neuro Oncol. 2018;20(suppl 4):1-86.

3. Whittle IR, Smith C, Navoo P, Collie D. Meningiomas. Lancet. 2004:363(9420):1535-1543.

4. Goldbrunner R, Minniti G, Preusser M, et al. EANO guidelines for the diagnosis and treatment of meningiomas. Lancet Oncol. 2016;17(9):e383-e391.

5. Van Alkemade H, De Leau M, Dieleman EMT, et al. Impaired survival and longterm neurological problems in benign meningioma. Neuro Oncol. 2012;14(5):658666.
6. Kondziolka D, Levy EI, Niranjan A, Flickinger JC, Dade Lunsford D. Longterm outcomes after meningioma radiosurgery: physician and patient perspectives. J Neurosurg. 1999;9(4):310.

7. Zamanipoor Najafabadi AH, Peeters MCM, Lobatto DJ, et al. Impaired healthrelated quality of life of meningioma patients-a systematic review. Neuro Oncol. 2017;17(9):897-907.

8. Jefford M, Karahalios E, Pollard A, et al. Survivorship issues following treatment completion-results from focus groups with Australian cancer survivors and health professionals. J Cancer Surviv. 2008;2(1):20-32.

9. Morgan MA. Cancer survivorship: history, quality-of-life issues, and the evolving multidisciplinary approach to implementation of cancer survivorship care plans. Oncol Nurs Forum. 2009;36(4):429-436.

10. Dijkstra M, van Nieuwenhuizen D, Stalpers LJ, et al. Late neurocognitive sequelae in patients with WHO grade I meningioma. J Neurol Neurosurg Psychiatry. 2009;80(8):910-915.

11. Goebel S, Mehdorn HM. Development of anxiety and depression in patients with benign intracranial meningiomas: a prospective long-term study. Support Care Cancer. 2013;21(5):1365-1372.

12. Douw L, Klein M, Fagel SS, et al. Cognitive and radiological effects of radiotherapy in patients with low-grade glioma: long-term follow-up. Lancet Neurol. 2009;8(9):810-818.

13. Spinhoven P, Ormel J, Sloekers PPA, Kempen G, Speckens AEM, Van Hemert AM. A validation study of the Hospital Anxiety and Depression Scale (HADS) in different groups of Dutch subjects. Psychol Med Copyr. 1997;27(2):363370.

14. van Roijen L, Essink-Bot ML, Koopmanschap MA, Bonsel G, Rutten FFH. Labor and health status in economic evaluation of health care. The health and labor questionnaire. Int J Technol Assess Heal Care. 1996;12(3):405-415.

15. Taphoorn MJ, Henriksson R, Bottomley A, et al. Health-related quality of life in a randomized phase III study of bevacizumab, temozolomide, and radiotherapy in newly diagnosed glioblastoma. J Clin Oncol. 2015;33(19):21662175 .

16. Osoba D, Rodrigues G, James M, Zee B, Joseph P. Interpreting the significance of changes in health-related quality-of-life scores. J Clin Oncol. 1998;16(1): 139-144.

17. Bjorner JB, Wallenstein G V, Martin MC, et al. Interpreting score differences in the SF-36 vitality scale: using clinical conditions and functional outcomes to define the minimally important difference. Curr Med Res Opin. 2007;23(4):731-739.

18. Edelvik A, Taft C, Ekstedt G, Malmgren K. Health-related quality of life and emotional well-being after epilepsy surgery: a prospective, controlled, long-term follow-up. Epilepsia. 2017;58(10):1706-1715.

19. van Boxtel MPJ, Buntinx F, Houx PJ, Metsemakers JFM, Knottnerus A, Jolles $\mathrm{J}$. The relation between morbidity and cognitive performance in a normal aging population. J Gerontol. 1998;53A(2):M147-M155.

20. Lezak MD, Howieson DB, Loring DW. Neuropsychological Assessment. New York: Oxford Univerity Press; 2004.

21. Centraal Bureau voor de Statistiek. Arbeidsdeelname; kerncijfers. https:// opendata.cbs.nl/statline/portal.html?_la=nl\&_catalog=CBS\&tableId= 83056NED\&_theme $=170$. Accessed June 4, 2018.

22. Pignatti BF, Van Den Bent M, Curran D, et al. Prognostic factors for survival in adult patients with cerebral low-grade glioma. J Clin Oncol. 2019;20(8):20762084.

23. Zamanipoor Najafabadi AH, Peeters MCM, Lobatto DJ, et al. Health-related quality of life of cranial WHO grade I meningioma patients: are current questionnaires relevant? Acta Neurochir (Wien). 2017;159(11):2149-2159.

24. Nassiri F, Price B, Shehab A, et al. Life after surgical resection of a meningioma: a prospective cross-sectional study evaluating health-related quality of life. Neuro Oncol. 2019;21(suppl 1):i32-i43.

25. Dirven L, Taphoorn MJ, Reijneveld JC, et al. The level of patient-reported outcome reporting in randomised controlled trials of brain tumour patients: a systematic review. Eur J Cancer. 2014;50(14):2432-2448.

26. Perrotti A, Ecarnot F, Monaco F, et al. Quality of life 10 years after cardiac surgery in adults: a long-term follow-up study. Health Qual Life Outcomes. 2019;17(1): $1-9$.

27. Ng CY, Ballantyne JA, Brenkel IJ. Quality of life and functional outcome after primary total hip replacement: a five-year follow-up. J Bone Jt Surg Ser B. 2007;89(7):868-873.

28. Rijnen S, Meskal I, Bakker M, Rutten G, Gehring K, Sitskoorn M. Cognitive outcomes in meningioma patients undergoing surgery: individual changes over 
time and predictors of late cognitive functioning. Neuro Oncol. 2018;20(suppl 3):112.

29. Bunevicius A, Deltuva VP, Tamasauskas A. Association of pre-operative depressive and anxiety symptoms with five-year survival of glioma and meningioma patients: a prospective cohort study. Oncotarget. 2017;8(34):57543-57551.

30. Jakola AS, Gulati M, Gulati S, Solheim O. The influence of surgery on quality of life in patients with intracranial meningiomas: a prospective study. $J$ Neurooncol. 2012;110(1):137-144.

31. Henzel M, Fokas E, Sitter H, Wittig A, Engenhart-Cabillic R. Quality of life after stereotactic radiotherapy for meningioma: a prospective non-randomized study. J Neurooncol. 2013;113(1):135-141.

32. van Nieuwenhuizen D, Klein M, Stalpers LJ, Leenstra S, Heimans JJ, Reijneveld JC. Differential effect of surgery and radiotherapy on neurocognitive functioning and health-related quality of life in WHO grade I meningioma patients. J Neurooncol. 2007;84(3):271-278.

33. Brastianos PK, Galanis E, Butowski N, et al. Advances in multidisciplinary therapy for meningiomas. Neuro Oncol. 2019;21(suppl 1):i18-i31.

\section{Acknowledgments}

We would like to acknowledge the research nurses of the LUMC/HMC Neurosurgery departmentfor their help with the data collection. Other collaborators of the Dutch Meningioma Consortium are acknowledged: Wouter A. Moojen, MD, PhD, University Neurosurgical Centre Holland, Leiden University Medical Centre, Haaglanden Medical Centre and Haga Teaching Hospital, Leiden and The Hague, the Netherlands; and Jaap C. Reijneveld, MD, PhD, Brain Tumor Center Amsterdam at Amsterdam University Medical Centers, location VUmc, Amsterdam, the Netherlands, and Department of Neurology, Amsterdam University Medical Centers, location VUmc, Amsterdam, the Netherlands

Supplemental digital content is available for this article at www. neurosurgery-online.com.

Supplemental Digital Content 1. Table. Outcome measures: questionnaires and neuropsychological test.

Supplemental Digital Content 2. Text. Sample size calculation and Rationale propensity score regression analysis.

Supplemental Digital Content 3. Table. Sociodemographic and clinical characteristics of meningioma patients stratified per treatment strategy.

Supplemental Digital Content 4. Table. Nonresponder analysis: comparing participating patients with patients who declined to participate in this study.

Supplemental Digital Content 5. Table. Raw SF-36 score and EORTS QLQBN20 scores.

Supplemental Digital Content 6. Propensity score adjusted differences between meningioma patients and controls for the SF-36 domains role limitations due to physical functioning and social functioning.

Supplemental Digital Content 7. Table. Propensity score adjusted differences between meningioma patients and controls for the SF-36 domains role limitations due to emotional functioning, vitality, and the mental component score.

Supplemental Digital Content 8. Table. Propensity score adjusted differences between meningioma patients and controls for the cognitive domains executive functioning, verbal memory, and working memory.

Supplemental Digital Content 9. Table. Propensity score adjusted differences between meningioma patients and controls for the cognitive domains of attention, information processing speed, and psychomotor functioning.

Supplemental Digital Content 10. Figure. Health-related quality of life scores for different treatment strategies.

Supplemental Digital Content 11. Figure. Neurocognitive functioning scores for different treatment strategies.

\section{COMMENTS}

The authors present their analysis of a multicenter, prospective, cross-sectional study to investigate the long-term disease burden of meningioma patients. Their goal was to describe the impact of meningioma treatment on patients' health-related quality of life (HRQoL) and neurocognitive function well after patients completed their treatment for their meningioma. Meningioma patients were added to this analysis if greater than 5 years had passed since their last meningioma intervention.

The studied patient cohort had a median 9 years since last intervention for their meningioma. The authors found that meningioma patients reported more limitations in physical and emotional abilities and were more anxious and depressed when compared with controls. Almost $50 \%$ of patients had neurocognitive deficits. Postoperative complications, use of radiotherapy, and reresection surgery were associated with worse neurocognitive functioning. Meningioma patients were less likely to be working than population controls. The authors conclude that meningioma patients suffer with severe disease burden despite "curative" resection.

While results in patient-reported outcome measures (PROMs) can be influenced by pre-existing neuropsychological comorbidities, HRQoL analyses are worthwhile studies for investigating the unforeseen toll upon patient wellbeing that neurosurgical management of "benign" pathology entails. ${ }^{1} \mathrm{HRQOL}$ analysis like this will increase our understanding of the long-term effects of the treatment of intracranial meningioma so we can better counsel and support our meningioma patients. Future papers will need to focus on the effect of preexisting neuropsychological comorbidities, meningioma location, and specific meningioma treatment to highlight at-risk populations.

David P. Bray Jeffrey J. Olson Atlanta, Georgia

1. Angevine PD, Bray D, Cloney M, Malone H. Uncertainty in the relationship between sagittal alignment and patient-reported outcomes. Neurosurgery. 2020;86(4):485-491

n this multicenter cross-sectional study, 190 patients were studied for at least five years after surgery and radiotherapy for intracranial meningiomas, including active MRI surveillance and serial assessment of health related quality of life (SF-36), neurocognitive functioning (neuropsychological assessment), anxiety and depression (HADS), and work productivity (SF-HLQ). At a median of nine years follow-up after intervention, patients reported more limitations due to physical and emotional functioning compared with controls. Patients also had an increased risk to suffer from anxiety and depression. Patients of working age less often had a paid job compared to controls. This study serves as an important reminder of the effect of meningioma treatment on quality of life and the importance of initial treatments being successful and durable given the finding that multiple treatments exert additive negative effects on quality of life. These are important findings for neurosurgeons treating meningioma patients.

Manish K. Aghi

San Francisco, California 